\title{
The Investigation of Icon Concreteness with and without Text Describe for Retirees
}

\author{
Kleddao Satcharoen* \\ Faculty of Engineering, King Mongkut's Institute of Technology Ladkrabang, Bangkok, Thailand. \\ * Corresponding author. Tel.: (66) 2329 8000; email: kleddao@gmail.com \\ Manuscript submitted December 10, 2018; accepted January 20, 2019. \\ doi: $10.17706 /$ jcp.14.2.119-124
}

\begin{abstract}
The purpose of this study was to examine icon design for retirees, focusing on icon concreteness. The study was undertaken because although older adults are increasingly likely to use computers, the Internet, and portable computing devices like smartphones and tablets, they still face usability challenges including cognitive and skill barriers. Previous research has shown that concrete icons increase selection accuracy for inexperienced users. This research was an experiment $(n=30$ ), in which users were asked to identify concrete and abstract icons. Chi-square analysis did not show a significant difference in selection accuracy of abstract and concrete icons in an unprompted trial $\left(\chi^{2}=1.493, \mathrm{df}=5, \mathrm{p}=.914\right)$ or a text-prompted trial $\left(\chi^{2}=.715\right.$, $\left.\mathrm{df}=3, \mathrm{p}=.870\right)$. However, results were significantly better for the prompted trial. The implication is that abstract and concrete icons are equally difficult for senior adults to recognize.
\end{abstract}

Key words: Icon concreteness, text describe, retirees, accuracy.

\section{Introduction}

On the global scale, computer and Internet use is becoming more common, though it is not yet ubiquitous As of December $2017,51 \%$ of the world's population had Internet access, although this access is not evenly distributed between global regions [1]. A global average of 38\% of adults have computers available in their households [2]. However, the biggest boost for computing power has been the emergence of mobile computing devices like tablets and smartphones, which are both cheaper and easier to use than fixed computers. As of 2015, there were an estimated 1.86 billion smartphone users worldwide, a figure which is expected to rise to 2.87 billion worldwide by 2020 [3]. However, this increasing computer and Internet access is not evenly spread. There is a long-standing digital divide, in which factors like age, education, socioeconomics, and location determine to some extent whether an individual will use computers and the Internet [4]. One element of the digital divide is age - older users have long been known to use computers and the Internet at a lower rate than younger users [5]. There is some evidence that this difference is weakening, with now only the oldest seniors showing significant usage differences [5]. However, senior users still face significant user interface issues with computers, which have not been explored in enough detail to optimize computers for their use. This study's purpose was to examine one small aspect of the computer usage needs of seniors - that of icon design, specifically icon concreteness 


\subsection{Computer Use and Elderly People}

At one time, elderly people on the wrong side of the so-called digital divide, with seniors in most countries not having the skills, tools, or even the need to use the Internet or computing technology [4]. Over time, this has changed, and now more and more seniors are using computers and the Internet [5]. As [5] analysis showed that Internet and computer use was not distributed evenly among seniors, with the very old (those aged 70+) still being significantly less likely to use computers and the Internet than younger seniors. In part, this is because younger seniors are more likely to have acquired computer and Internet skills in the workplace and to have personal and working environments where such use is encouraged [6]. Thus, while computer use among elderly people has risen dramatically in the past several years [5], there are still groups that may have less access than others.

Seniors face many challenges in using computers and the Internet. For example, they may experience frustration and learning challenges when using computers, or may have cognitive or physical functional problems that prevent effective computer use [7]. The interface design of a computing system can also pose challenges for seniors, as interface elements like icons and menus can be hard to perceive, understand and navigate [8]. Navigation issues from poor icon design can seriously impede system usability by seniors, as found by several studies [9]-[11]. However, these studies have mainly focused on specific computing contexts and programs rather than examining icon design preferences.

Computer use is still an important skill for the elderly, particularly since it can help them maintain social and personal connections, access resources, and perform other important tasks [7]. However, to be accessible to seniors, there is a need to consider issues like icon design to meet their needs more broadly than previously studied.

\subsection{Icon Design}

An icon is a visual tool used to provide easy interaction with a computing system such as a computer, tablet, or mobile phone, performing a single pre-defined command such as launching a program when it is used [12]. The icon is one of the oldest elements of the graphic user interface (GUI) and is used in most modern computing systems [12].

The icon design characteristic studied here is abstractness and concreteness. An abstract icon is one that is not a direct visual representation of the action or tool it is associated with, while a concrete icon is one that does have a direct visual representation of its underlying action [13]. Abstractness and concreteness are a spectrum, rather than independent characteristics, and they have different proposed mechanisms for action [14]. For example, while abstract icons are visually simpler and therefore easier to recognize, concrete icons have a stronger connection to stereotypical meanings and can be more easily puzzled out by inexperienced users [14]. Thus, early research provided conflicting answers on whether icons should be designed as abstract or concrete. However, more recent studies have suggested that concrete icons are more easily recognized [15]. These authors showed that icon concreteness was positively correlated to selection accuracy, and that this effect was particularly strong for users unfamiliar with the system. Thus, concrete rather than abstract icon design is a better choice for accuracy, especially when designing for inexperienced users [15]. Furthermore, a cross-cultural study showed that people from both Western and Eastern cultures (including mainly Europeans and Thai people) had a strong preference for concrete icons, indicating that this preference may be widespread if not entirely universal [16]. One area where there is a gap in the research is the preference for abstract or concrete icons among the elderly. One review of mobile interfaces showed that older adults may prefer concrete icons due to lack of familiarity and cognitive 
limitations [17]. However, more evidence is needed to determine whether there is a significant performance difference.

\section{Methodology}

The study was conducted using an experimental approach. The sample was selected from respondents to a study recruitment notice. Selection criteria included that the respondents were retired and that they did not have any significant hearing or visual impairments and were not color blind. A total of 30 participants were selected. All participants were Thai speakers and aged over 60. The gender distribution was approximately equal ( $\mathrm{n}=16$ men, $\mathrm{n}=14$ women).

The study was conducted in a well-lit fixed environment. The experimental apparatus included a desktop computer and a selection of icons from common computing environments, including OS X, Microsoft Windows, and Internet-based icons. Microsoft Visual Basic Programming was used to produce the stand alone software for participant to do the test. For the experiment in the stand alone software, the icons were arranged in a circle around the center of the screen. The circular array included one target icon and seven distractors.

Respondents were asked to do two trials, with 10 icons in each trial (five concrete icons and five abstract icons). In both trials, the selection accuracy was the target outcome. The icons were randomly selected from a prepared database. For the first trial, respondents were verbally prompted with the name of one of the icons and asked to select it on the screen. For the second trial, a brief text was displayed below the icons that explained its function. The respondents were again verbally prompted to select a specific icon. In each trial, the number of accurate responses were logged separately for the concrete and abstract icon categories.

SPSS (Statistical Package for the Social Sciences) version 23 was used to analyze the data from the experiment. Results were analyzed using distribution tables and chi-square tests to determine if there was any significant difference in distribution between abstract and concrete icons. The chi-square was accepted as a distribution difference at $\mathrm{p}<.05$.

\section{Results}

\subsection{Trail 1: No Text Prompt Condition}

Table 1. Distribution of Correct Responses in Trial 1 (no Text Prompt Condition)

\begin{tabular}{lcccc}
\hline \multirow{2}{*}{ Answer } & \multicolumn{2}{c}{ Abstract } & \multicolumn{2}{c}{ Concreteness } \\
\cline { 2 - 5 } & Frequency & Percent & Frequency & Percent \\
\hline All answer were wrong & 8 & 26.7 & 7 & 23.3 \\
1 correct & 11 & 36.7 & 14 & 46.7 \\
2 correct & 6 & 20.0 & 4 & 13.3 \\
3 correct & 2 & 6.7 & 2 & 6.7 \\
4 correct & 1 & 3.3 & 2 & 6.7 \\
All answer were correct & 2 & 6.7 & 1 & 3.3 \\
\hline Total & 30 & 100 & 30 & 100.0 \\
\hline
\end{tabular}

In the first trial, there was no text prompt offered for the participants to match; instead, they relied on the visual image of the icon only. Results are shown in Table 1. As this shows, accuracy rates were low, with most respondents getting no or one icons right for both abstract (63.3\%) and concrete (70\%) icon groups. $10 \%$ of respondents got four or five icons correct for both abstract and concrete icons. This suggests that 
without the text prompt, there are similar accuracy conditions for both abstract and concrete icons. A chi-square distribution test confirms this similarity $\left(\chi^{2}=1.493, \mathrm{df}=5, \mathrm{p}=.914\right)$.

\subsection{Trail 2: Text Prompt Condition}

In the second trial, a brief text prompt explaining the icon was included underneath the icon. Unsurprisingly, as Table 2 shows, accuracy rates for this trial were much higher. Most respondents scored four or five responses accurate for both the abstract group (90\%) and the concrete group (83.3\%). In this trial, no respondents got zero or one responses correct. Thus, inclusion of the text dramatically improved recognition of the icon between respondents. However, once again a chi-square distribution test shows that there was no significant difference between abstract and concrete icons $\left(\chi^{2}=.715, \mathrm{df}=3, \mathrm{p}=.870\right)$

Table 2. Distribution of Correct Responses in Trial 2 (Text Prompt Condition)

\begin{tabular}{lcccc}
\hline \multirow{2}{*}{ Answer } & \multicolumn{2}{c}{ Abstract } & \multicolumn{2}{c}{ Concreteness } \\
\cline { 2 - 5 } & Frequency & Percent & Frequency & Percent \\
\hline 2 correct & 1 & 3.3 & 2 & 6.7 \\
3 correct & 2 & 6.7 & 3 & 10.0 \\
4 correct & 12 & 40.0 & 10 & 33.3 \\
All answer were correct & 15 & 50.0 & 15 & 50.0 \\
\hline Total & 30 & 100 & 30 & 100.0 \\
\hline
\end{tabular}

\section{Discussion}

This research was undertaken because of the lack of research on icon design for senior users. Concreteness of a computing icon is one of the fundamental characteristics that helps users, especially inexperienced users, identify the purpose of the icon and effectively select it [14]. Furthermore, there are potential advantages to both abstract and concrete icons: while abstract icons are easier to identify visually, concrete icons have a shorter semantic distance, offering an advantage to inexperienced users [14]. Thus, this characteristic was selected as a basic functional characteristic that could influence how well senior users could use computers and what level of frustration they would experience. However, the results did not show that the concreteness of an icon made a significant difference in the effectiveness of selection. Instead, abstract and concrete icons were identified at similar rates in both trials. The main difference is that in the trial that used a text prompt, users had much higher identification rates. This suggests that for senior users, it is not abstractness or concreteness that matters for visual identification, but instead availability of external information.

These results were different from those found in previous studies, which have generally identified concrete icons as preferable to abstract icons for accuracy (though not necessarily reaction time) [14]-[16]. However, the results are not necessarily surprising given the nature of these findings and the role of experience in selection accuracy. As one study pointed out, a preference for icon concreteness is particularly strong among inexperienced users and those with cognitive or physical deficits that reduce function [17]. In earlier years, this may have have been true for older adults, who were not as skilled at using digital technology [4]. However, in recent years, older adults and seniors have become increasingly familiar with computing technology and willing to use it [5]. Thus, the effect of concreteness as it relates to usability for older adults may have faded.

There are still other problems that could affect individual preferences for abstract or concrete icons that need to be considered; for example, individuals could suffer cognitive or physical issues that make icon 
recognition hard [7] or may have physical limitations such as visual impairments that affect their ability to use such systems [8]. This study did not examine individual-level performance or cognitive deficits, so cannot speak to those limitations. However, the findings do agree that rather than being concerned with abstractness or concreteness as a primary point of icon design, there should be more attention paid to text prompts and other external meaning cues [12].

\section{Conclusion and Recommendations}

This research examined the effect of the abstractness or concreteness of an icon on selection accuracy for retirees. The experimental set-up used two different trials: an unprompted trial, and a trial in which a brief visual prompt was provided alongside the icon. Unexpectedly, the results did not show a significant difference between the abstract and concrete icons in terms of selection accuracy in either trial. However, they did show that there was a major difference in selection accuracy between the unprompted and prompted trials. In the unprompted trial, between $63 \%$ and $70 \%$ of respondents got no more than one answer correct in each category. In the prompted trial, between $83 \%$ and $90 \%$ got four to five answers correct in each category.

The findings show that providing text prompts could significantly improve selection accuracy for senior users. The results do not provide evidence for increased usability for either abstract or concrete icons. Based on prior evidence [15], it is probably still preferable to use concrete icons, particularly for inexperienced user groups such as older seniors. However, this may be most effective in conjunction with a brief text prompt. Thus, the recommendation is to use both text prompts and concrete icons when designing for seniors

There are some limitations to this study. The sample was relatively small $(n=30)$ and all respondents included were Thai, which could mean that there are cultural variances that were not observed. The study also did not consider factors like previous experience with computers, which could affect individual selection accuracy and preference for abstract and concrete icons. These gaps provide areas for further research, especially focused on usability of icons for seniors

\section{Acknowledgment}

I would like to thanks all the participants for participating in this study. I appreciate your honesty and willingness to assist with this important research.

\section{References}

[1] Miniwatts marketing group. (2018). Internet Usage Statistics: The Internet Big Picture. Retrieved from internet world stats: http://www.internetworldstats.com/stats.htm

[2] Pew research center. (2015, March 19). Global Computer Ownership. Retrieved from pew research center:

http://www.pewglobal.org/2015/03/19/internet-seen-as-positive-influence-on-education-but-negative -influence-on-morality-in-emerging-and-developing-nations/technology-report-15/

[3] Statista. (2018). Number of Smartphone Users Worldwide from 2014 to 2020 (in Billions). Retrieved from Statista: https://www.statista.com/statistics/330695/number-of-smartphone-users-worldwide/

[4] Chinn, M. D., \& Fairlie, R. W. (2007). The determinants of the global digital divide: A cross-country analysis of computer and internet penetration. Oxford Economic Papers, 59(10), 16-44.

[5] Friemel, T. N. (2016). The digital divide has grown old: Determinants of a digital divide among seniors. 
New Media and Society, 18(2), 313-331.

[6] Wagner, N., Hassanein, K., \& Head, M. (2010). Computer use by older adults: A multi-disciplinary review. Computers in Human Behavior, 26, 870-882.

[7] Gatto, S. L., \& Tak, S. H. (2008). Computer, internet adn email use among older adults: Benefits and barriers. Educational Gerontology, 34, 800-811.

[8] Liu, S., \& Joines, S. (2012). Developing a framework of guiding interface design for older adults. Proceedings of the Human Factors and Ergonomics Society Annual Meeting: Vol. 56 (pp. 1967-1971). Los Angeles, CA: Sage Publications.

[9] Azir, R. N., Maksom, Z., \& Naim, C. P. (2014). Tackling design issues on elderly smartphone interface design using activity centered design approach. ARPN Journal of Engineering and Applied Sciences, 9, 1190-1196.

[10] Li, Q., \& Yan, L. (2016). Older adults and digital technology: A study of user perception and usage behavior. Advances in Physical Ergonomics and Human Factors, 155-163.

[11] Yueh, H. P., Lin, W., Lu, T. Y., \& Chou, Y. L. (2013). Examining older users' performance on and preference for menu designs of digital photo frames. Theoretical Issues in Ergonomics Science, 14(3), 273-289.

[12] Preece, J., Sharp, H., \& Rogers, Y. (2015). Interaction Design: Beyond Human-Computer Interaction (4th ed.). Hoboken, NJ: John Wiley and Sons.

[13] Lin, R. (1994). A study of visual features for icon design. Design Studies, 15(2), 185-197.

[14] McDougall, S. J., Curry, M. B., \& de Bruijn, O. (1999). Measuring symbol and icon characteristics: Norms for concreteness, complexity, meaningfulness, familiarity, and semantic distance for 239 symbols. Behavior Research Methods, Instruments and Computers, 31(3), 487-519.

[15] McDougall, S., \& Isherwood, S. (2009). What's in a name? The role of graphics, functions and their interrelationships in icon identification. Behavior Research Methods, 41(2), 325-336.

[16] Chanwimalueng, C., \& Rapeepisarn, K. (2013). A study of the recognitions and preferences on abstract and concrete icon styles on smart phone from Easterners and Westerners' point of view. Proceedings of 2013 International Conference on Machine Learning and Cybernetics (ICMLC): Vol. 4 (pp. 1613-1619). New York: IEEE.

[17] Punchoojit, L., \& Hongwarittorrn, N. (2017). Usability studies on mobile user interface design patterns: A systematic literature review. Advances in Human-Computer Interaction.

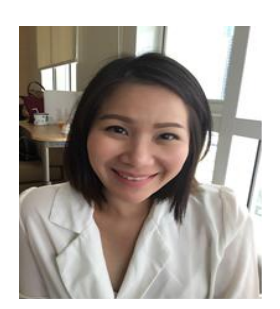

Kleddao Satchaoren was born in May 1978, Thailand. She earned her DPhil in computing (2017) and MSc in computing (2003) from the University of Buckingham, UK, and a MA in political science (2005) from Bangkok, Thailand. She also holds a BS.c in management technology (2000) from King Mongkut's Institute of Technology Ladkrabang, Bangkok, Thailand.

Since 2004, she has been working as the lecturer in Faculty of Engineer, Department of Computer Engineering at King Mongkut's Institute of Technology Ladkrabang, Bangkok, Thailand. She currently teaches 3 main courses: human computer interaction, object oriented analysis and design, and web technology. Therefore, her interest is on human computer interaction area, specific in icon characteristics, perception and algorithmic/programmatic techniques for icon characteristics measurement. 